\title{
Storage and Retrieval of a Microwave Field in a Spin Ensemble
}

\author{
Y. Kubo ${ }^{1}$, I. Diniz ${ }^{2}$, A. Dewes ${ }^{1}$, V. Jacques ${ }^{3}$, A. Dréau ${ }^{3}$, J.-F. \\ Roch $^{3}$, A. Auffeves ${ }^{2}$, D. Vion ${ }^{1}$, D. Esteve ${ }^{1}$, and P. Bertet ${ }^{1}$ \\ ${ }^{1}$ Quantronics group, SPEC (CNRS URA 2464), IRAMIS, \\ DSM, CEA-Saclay, 91191 Gif-sur-Yvette, France \\ ${ }^{2}$ Institut Néel, CNRS, BP 166, 38042 Grenoble, France and \\ ${ }^{3}$ LPQM (CNRS UMR 8537), ENS de Cachan, 94235 Cachan, France
}

(Dated: October 26, 2018)

\begin{abstract}
We report the storage and retrieval of a small microwave field from a superconducting resonator into collective excitations of a spin ensemble. The spins are nitrogen-vacancy centers in a diamond crystal. The storage time of the order of $30 \mathrm{~ns}$ is limited by inhomogeneous broadening of the spin ensemble.
\end{abstract}

Superconducting qubits are promising candidates for quantum information processing; however their coherence times [1] cannot yet compete with those of microscopic systems such as atoms [2, electron or nuclear spins [3]. Hybrid quantum circuit architectures have thus been proposed [4] 8], in which microscopic systems would be used as quantum memory for superconducting qubits. Whereas the coupling of one individual atom or spin to a superconducting circuit is usually too weak, the coupling constant of an ensemble of $N$ such systems is enhanced by $\sqrt{N}$, allowing to reach the strong coupling regime requested for quantum information applications. Proposals for spin-ensemble based hybrid quantum circuits often consist of a superconducting resonator used as a quantum bus between the ensemble and the superconducting qubit. On the experimental side [9-12, strong coupling between a spin ensemble and a superconducting resonator has up to now been demonstrated only spectroscopically. Here we report the first time-domain measurements of the coherent storage and retrieval of a classical microwave field [13] of about 100 photons from a superconducting resonator into collective excitations of a spin ensemble consisting of negatively-charged nitrogenvacancy centers in diamond (NV centers), an important step towards a spin-based hybrid quantum circuit architecture.

The experiment relies on the fact that the interaction between the electromagnetic field in the cavity mode and the spin ensemble involves only one collective spin variable, which behaves as a harmonic oscillator in the limit of low excitation energy [8]. This effective spin oscillator is magnetically coupled with a collective coupling constant $g_{\text {ens }}$ to the superconducting resonator whose frequency can be tuned. When two such coupled harmonic oscillators are suddenly put into resonance, they coherently exchange energy with a period $\pi / g_{\text {ens }}$. We observe this dynamics by measuring the amplitude of the microwave field leaking out of the resonator after its interaction with the spins, which is found to oscillate as a function of the interaction time. This storage-retrieval cycle is however damped in a relatively short time, which as we show is limited by the inhomogeneous broadening of the NV center ensemble. Quantitative agreement with recent theoretical work [14, 15] is obtained for a consis- tent set of data, covering spectroscopic as well as timedomain measurements.

In our experiment [9] (see Fig 1/ and Supplementary Material), an ensemble of $\sim 10^{12} \mathrm{NV}$ centers in a diamond crystal are magnetically coupled to a superconducting resonator. A static magnetic field $B_{\mathrm{NV}}=1.7 \mathrm{mT}$ is applied to the spins, parallel to the chip surface, along the $[1,0,0]$ crystal axis within a few degrees (see Fig $1 \mathrm{p}$ ). With this orientation, the four possible NV center crystalline orientations all make approximately the same angle $\theta \simeq 55^{\circ}$ with $B_{\mathrm{NV}}$ so that their resonance frequencies (see Fig 1 ) are approximately equal. The resonator includes a four-SQUID array so that its frequency $\omega_{\mathrm{r}}(\Phi)$ can be tuned by changing the flux $\Phi$ through the SQUIDs loop [16. Such a frequency tuning can be done on a nanosecond timescale [17. For time-domain experiments, we measure the amplitude $A(t)$ of microwave pulses transmitted through the resonator using homodyne detection followed by sampling and averaging.

We first characterize the resonator-spins system by measuring the resonator transmission while scanning $\Phi$. As shown in Fig,11, two vacuum Rabi splittings are observed when the resonator frequency matches either of the two NV center transitions $\omega_{-}$(resp. $\omega_{+}$) from ground state $m_{\mathrm{S}}=0$ to $m_{\mathrm{S}}=-1\left(\right.$ resp. $\left.m_{\mathrm{S}}=+1\right)$. A fit of these data using a coupled oscillators model 9 yields $g_{\text {ens }} / 2 \pi=10.6 \mathrm{MHz}$ for the lower frequency anticrossing occurring at $\omega_{-} / 2 \pi=2.85 \mathrm{GHz}$, on which we will focus in the following. The transmission close to the middle of the anticrossing is shown in Fig. 11 where two wellresolved polaritonic peaks can be seen, an indication that the two oscillators are in the strong coupling regime and that the coherent exchange of excitations between the resonator and the spin ensemble can be observed in the time-domain.

To demonstrate such dynamics, the experiment proceeds as follows (see Fig 2a). At the beginning of each experimental sequence (averaged out typically $5 \cdot 10^{5}$ times), the resonator is biased at a flux $\Phi_{\text {OFF }}$ such that its frequency $\omega_{\mathrm{r}}\left(\Phi_{\mathrm{OFF}}\right) / 2 \pi=2.88 \mathrm{GHz}$ is out of resonance with the spins. A microwave pulse at frequency $\omega_{\mathrm{r}}\left(\Phi_{\mathrm{OFF}}\right)$ of duration $2 \mu \mathrm{s}$ (much longer than the resonator damping time $\left.T_{\text {cav }}=Q / \omega_{\mathrm{r}} \sim 100 \mathrm{~ns}\right)$ establishes a steady-state coherent field of amplitude $\alpha$ inside 
(a)
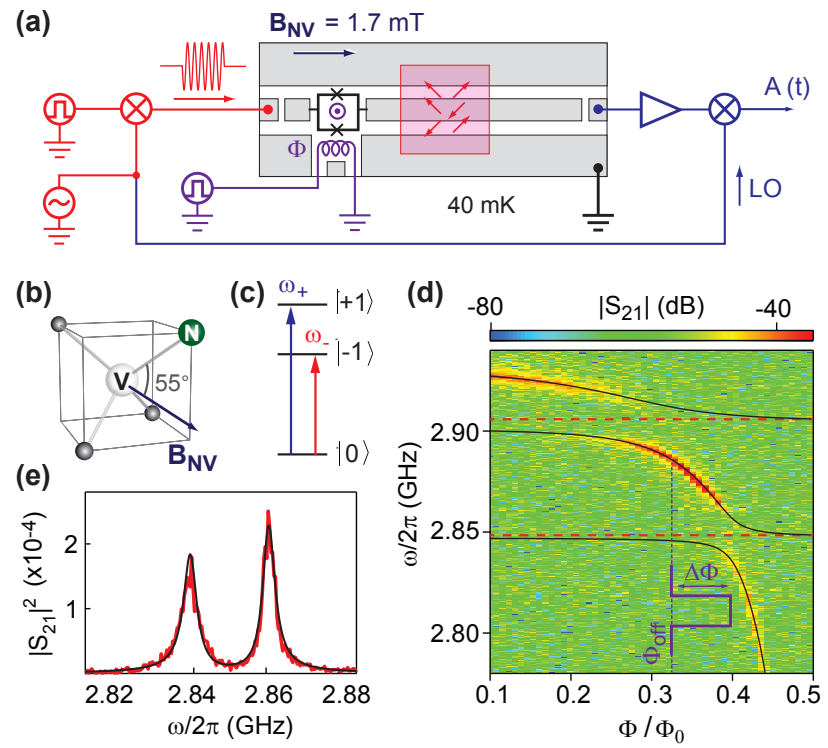

Figure 1: (a) Setup description. The NV centers ensemble is magnetically coupled to the coplanar resonator containing a SQUID. The flux $\Phi$ through the SQUID loop can be tuned on a nanosecond scale by applying current pulses to an on-chip antenna. A magnetic field $B_{\mathrm{NV}}=1.7 \mathrm{mT}$ is applied to the spins, parallel to the sample and to the $[1,0,0]$ crystal axis (b). The amplitude $A(t)$ of microwave pulses transmitted through the resonator is measured by homodyne detection at roomtemperature after amplification. (c) Simplified energy level scheme of a NV center. (d) Resonator transmission $\left|S_{21}\right|(\omega)$ at $B_{\mathrm{NV}}=1.7 \mathrm{mT}$ as a function of $\Phi$ (in units of the superconducting flux quantum $\Phi_{0}=h / 2 e$ ) showing two vacuum Rabi splittings. The solid line is a fit to the data using the coupled oscillators model [9]. (e) Vacuum Rabi splitting close to resonance with $\omega_{-}$. Black line is experimental data, red line is theory as explained in the text (rescaled in amplitude to fit the data), assuming a spins-resonator detuning of $0.5 \mathrm{MHz}$.

the resonator (with $|\alpha|^{2} \sim 120$ photons). Right after the microwave pulse is switched off $(t=0)$, the resonator frequency is brought close to $\omega_{-}$by a flux pulse of amplitude $\Delta \Phi$ (see Supplementary Material) and duration $\tau$, during which the resonator and the spin ensemble may exchange energy causing the intraresonator field amplitude $|\alpha(t)|$ to oscillate. After the flux pulse, the only evolution of the field is an exponential decay $|\alpha(t>\tau)|=|\alpha(\tau)| \exp \left(-(t-\tau) / 2 T_{\text {cav }}\right)$. Measuring the amplitude of the exponentially damped microwave signal that leaks out of the cavity therefore directly yields $|\alpha(\tau)|$ and reveals the coupled resonator-spins dynamics.

Figure 2 $\mathrm{b}$ shows the results obtained for a flux pulse amplitude $\Delta \Phi$ that puts the resonator in resonance with the spins at $\omega_{-}$. The two-dimensional plot shows the measured microwave output amplitude $A(t)$ for increasing $\tau$. The curve $A(t)$ for the shortest flux pulse $\tau=5 \mathrm{~ns}$ (see Fig. 2k) showing the microwave field decay after the pulse is switched off is only approximately exponential, due to a slight nonlinearity of the resonator caused by the presence of the SQUID [18, which we neglect in the fol- (a)
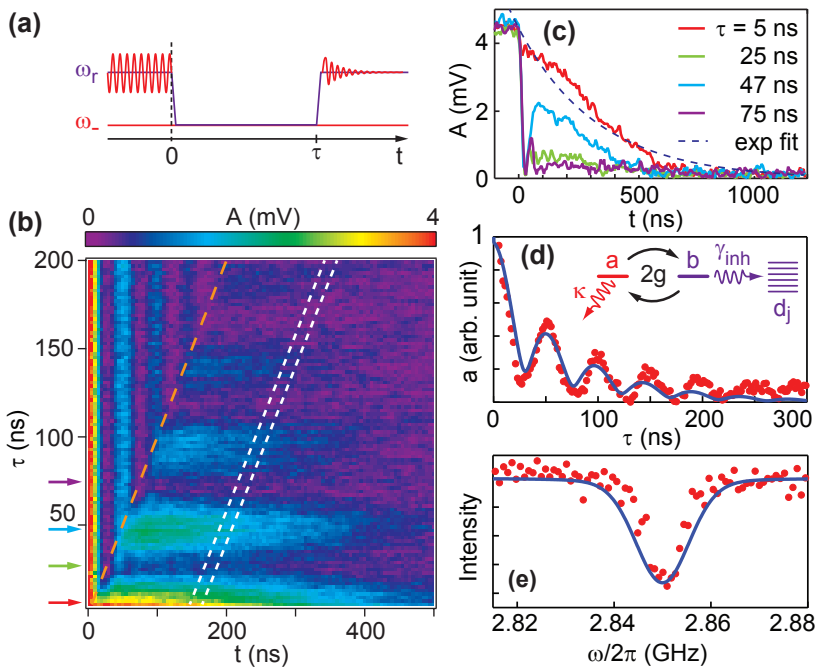

Figure 2: (a) Sketch of an experimental sequence (b) Output amplitude $A(t)$ measured for increasing flux pulse duration $\tau$, for a flux pulse amplitude such that $\omega_{r}\left(\Phi_{\text {OFF }}+\overline{\Delta \Phi}\right)=\omega_{-}$. The dashed orange line indicates $t=\tau$. The two white dashed lines indicate the time window on which averaging is performed to compute $a(\tau)$ (see text). (c) Amplitude $A(t)$ for $g_{\text {ens }} \tau=0, \pi, 2 \pi, 3 \pi$. (d) Normalized amplitude $a(\tau)=\overline{A\left(\tau+t_{\text {off }}\right)}$ (see text). Red dots are experimental data, continuous blue line is theory as explained in the text. (inset) Sketch of the physical process leading to damping of the oscillations because of coupling of the superradiant state to the bath of subradiant states. (e) ODMR signal measured at room-temperature (red dots), compared to the spin distribution $\rho(\omega)$ (see text) used in the theoretical calculations (blue line).

lowing. For increasing $\tau$, the curves $A(t)$ clearly display two parts : from $t=0$ to $t=\tau$ rapid transient oscillations are observed, which are unfortunately difficult to quantitatively interpret due to the finite bandwidth of our homodyne detection setup. After $t=\tau, A(t)$ shows a decay similar to the one observed for the shortest pulse but with an amplitude that oscillates with $\tau$ with a pe$\operatorname{riod} T \sim 50 \mathrm{~ns}$, in quantitative agreement with the coupling strength estimated from the vacuum Rabi splitting $T \simeq \pi / g_{\text {ens }}$. This establishes that the observed oscillation is indeed due to cycles where the microwave field is first stored into a collective spin excitation $\left(0<g_{\text {ens }} \tau<\pi\right)$ then retrieved $\left(\pi<g_{\text {ens }} \tau<2 \pi\right)$. Curves corresponding to various steps of the cycle are also shown in Fig. 2 . Note that by using a homodyne detection scheme we can only measure a field that has a well-defined phase relation with the local oscillator, implying that phase coherence is indeed preserved even after several (storage, retrieval) cycles. A full quantum state tomography would however be needed in order to assess the fidelity of the field storage at the quantum level, which is beyond the scope of the present work.

In order to obtain more quantitative insight into these data, we compute for each $\tau$ the quantity $a(\tau)=$ 
$\overline{A\left(\tau+t_{\text {off }}\right)}$ where $t_{\text {off }}=140 \mathrm{~ns}$ is an offset that was chosen to avoid taking into account transients and where an additional time-averaging is performed on a $20 \mathrm{~ns}$ window (see Fig. 2b). Since $a(\tau)$ is directly proportional to $|\alpha(\tau)|$ it is a good quantity to investigate the spin-resonator dynamics. It is plotted in Fig $2 \mathrm{~d}$ for the previous flux pulse parameters. Several cycles are observed before the oscillation amplitude and offset decay simultaneously to zero. The fact that the field amplitude decays to zero within a hundred of nanoseconds might come as a surprise given the fact that the energy damping time of individual spins is in the millisecond range at room-temperature and is expected to be well above one second at low temperature [11. The only energy dissipation should thus be caused by damping of the resonator, which occurs on a significantly longer timescale.

Understanding this decay requires to take into account the inhomogeneous broadening of the spin ensemble. Following [14, 15], we model each spin in the HolsteinPrimakoff approximation (valid in our experiment which is deep in the low-excitation regime) by a harmonic oscillator of frequency $\omega_{\mathrm{j}}$ with annihilation (creation) operator $b_{\mathrm{j}}\left(b_{\mathrm{j}}^{\dagger}\right)$, the resonator field being described by the annihilation (creation) operator $a\left(a^{\dagger}\right)$. The system Hamiltonian is then $H / \hbar=\omega_{\mathrm{r}}(\Phi) a^{\dagger} a+\sum \omega_{\mathrm{j}} b_{\mathrm{j}}^{\dagger} b_{\mathrm{j}}+\sum g_{\mathrm{j}}\left(b_{\mathrm{j}}^{\dagger} a+b_{\mathrm{j}} a^{\dagger}\right)$, $g_{\mathrm{j}}$ being the coupling constant of spin $j$ with the resonator. Turning to a new orthogonal basis consisting of the superradiant spin-wave mode $b=\left(1 / g_{\mathrm{ens}}\right) \sum g_{\mathrm{j}} b_{\mathrm{j}}$ of frequency $\omega_{-}$and $N-1$ orthogonal dark modes $d_{\mathrm{j}}$ of frequency $\tilde{\omega}_{\mathrm{j}}$, with $g_{\text {ens }}=\left(\sum\left|g_{\mathrm{j}}\right|^{2}\right)^{1 / 2}$, the Hamiltonian becomes $H / \hbar=\omega_{\mathrm{r}}(\Phi) a^{\dagger} a+\omega_{-} b^{\dagger} b+g_{\text {ens }}\left(a^{\dagger} b+h . c\right)+$ $\sum \tilde{\omega}_{\mathrm{j}} d_{\mathrm{j}}^{\dagger} d_{\mathrm{j}}+\sum \gamma_{\mathrm{j}}\left(b^{\dagger} d_{\mathrm{j}}+h . c\right)$ with $\gamma_{\mathrm{j}} \sim \Delta / \sqrt{N}$. Here $\Delta$ is the variance of the $\omega_{j}$ distribution, i.e. the inhomogeneous spins linewidth. Note that in this expression we have neglected terms coupling the dark modes together. From the previous expression we see that the superradiant spin-wave mode is the only one coupled to the resonator field (with constant $g_{\text {ens }}$ ), but that in the presence of inhomogeneous broadening $(\Delta>0)$ it is also coupled to the $N-1$ dark modes. In the large- $N$ limit, these dark modes act as a bath into which energy is damped at a rate $\gamma_{\text {inh }}$, explaining the fast decay in Fig $2 \mathrm{~d}$. Introducing the normalized spin density distribution $\rho(\omega)$ (centered around 0 instead of $\omega_{-}$), one can show that $\gamma_{\text {inh }} \simeq 2 \pi \rho\left(g_{\text {ens }}\right) g_{\text {ens }}^{2}$ [15 in the $g_{\text {ens }} \gg \Delta$ limit. An important consequence of this formula is that increasing $g_{\text {ens }}$ (by e.g. increasing the spins density) actually helps reducing $\gamma_{\text {inh }}$ provided $\rho(\omega)$ decays faster than $\omega^{-2}$ [14, 15]. This implies that a strong coupling to the cavity protects the system against the consequences of inhomogeneous broadening, an effect called cavity protection in [15. In our experiment however we are not in the $g_{\text {ens }} \gg \Delta$ limit (see below) and these approximate formula are not valid. We thus resort to explicit analytical formula obtained using input-output relations on $H$ [15], which allow us to calculate both the resonator transmission $t(\omega)=\kappa /\left[2 i\left(\omega-\omega_{\mathrm{r}}\right)-\kappa-2 i W(\omega)\right]$

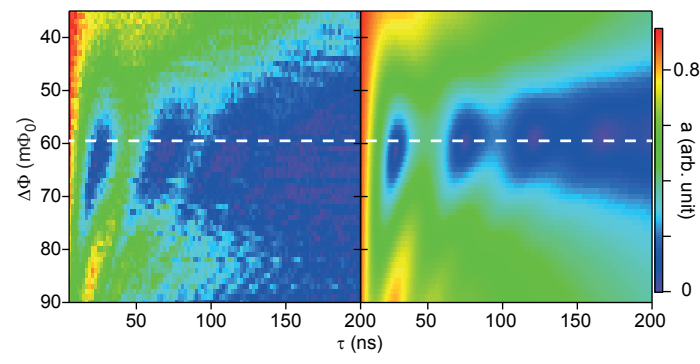

Figure 3: Coherent field exchange between the resonator and the spins for varying flux pulse amplitude $\Delta \Phi$. Normalized amplitude $a(\tau)$ (see text) is plotted versus $\tau$ and $\Delta \Phi$. Left graph is experimental data, right graph is theory. The white dashed line indicates $\overline{\Delta \Phi}$.

with $W(\omega)=g_{\text {ens }}^{2} \int_{-\infty}^{+\infty} \rho\left(\omega^{\prime}\right) d \omega^{\prime} /\left[\omega-\omega^{\prime}\right]$ and the timedomain signal as the modulus of the inverse FourierLaplace transform of $t(\omega)$ (see [15] and Supplementary Material).

To estimate these quantities in our experiment, we need to know the spin density distribution $\rho(\omega)$. In $\mathrm{NV}$ centers ensembles, the inhomogeneous linewidth is caused by dipolar interactions with neighboring spins, either paramagnetic nitrogen impurities (so-called P1 centers) that were not converted into NV centers during the sample processing, or ${ }^{13} \mathrm{C}$ nuclei that are present in natural abundance (1.1\%) [19]. Note that neighboring NV centers do not contribute to the inhomogeneous linewidth because at the temperature of our experiment they are frozen in the $m_{\mathrm{S}}=0$ state. In our sample, a FWHM linewidth $\Delta / 2 \pi \sim 7 \mathrm{MHz}$ was measured by optically-detected magnetic resonance (ODMR) at room-temperature (see Fig.2e), compatible with the linewidth expected from its nominal P1 centers concentration $\sim 100 \mathrm{ppm}[19$. As shown in [14, 15], quantitative predictions for the system dynamics require to know not only the overall linewidth but also the detailed shape of $\rho(\omega)$. In particular Gaussian and Lorentzian distributions yield very different results. Spin ensembles inhomogeneously broadened by dipolar interactions are expected to show a Lorentzian lineshape [20] with a cutoff; however complications in our experiment arise due to hyperfine coupling with the ${ }^{14} N$ atom nuclear spin, and to a possible misalignment of $B_{\mathrm{NV}}$ with the [1,0,0] crystalline axis causing the four NV orientations to have slightly different frequencies. As a result, we assume a phenomenological lineshape for our spin ensemble adjusted for reaching good agreement both with spectral and time-domain experimental data, as a convolution of a Gaussian and a Lorentzian profile with respective widths $\sigma$ and $\gamma$. The parameters chosen in the following $(\sigma / 2 \pi=5.12 \mathrm{MHz}$ and $\gamma / 2 \pi=1 \mathrm{MHz}$ ) yield a lineshape $\rho(\omega)$ compatible with ODMR data although slightly broader, as can be seen in Fig 2 e (see Supplementary Material). Using such distribution and the formulas above, we obtain quantitative agreement for spectroscopic (see Fig. 1 1 $)$ as well as time-domain (see Fig. 2d) measurements. 


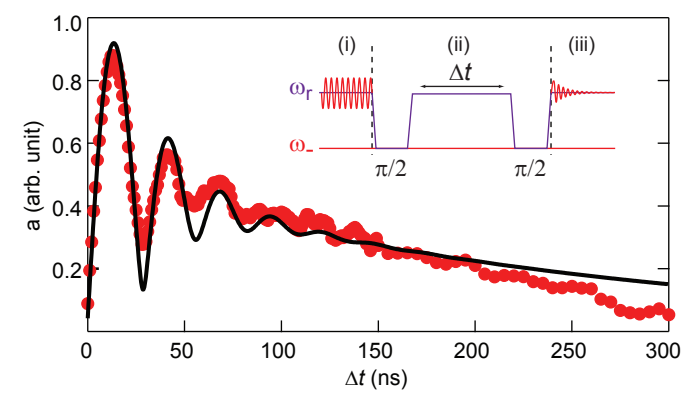

Figure 4: Ramsey fringes experiment. The normalized microwave amplitude $a(\Delta t)$ after the pulse sequence shown in inset is plotted. Red dots are experimental data, black continuous line is theory.

We also study the dependence of the microwave field exchange on the resonator-spins detuning by measuring $a(\tau)$ for various $\Delta \Phi$ (see Fig 3 . Out of resonance, the oscillations amplitude is reduced and their frequency increases as expected for two coupled oscillators. The asymmetry in the data between the $\Delta \Phi<\overline{\Delta \Phi}$ and $\Delta \Phi>\overline{\Delta \Phi}$ sides is an artefact of the nonlinear dependence of $\omega_{\mathrm{r}}$ on $\Delta \Phi$ and of a residual hybridization of the resonator with the spins caused by the finite initial detuning $\left[\omega_{r}\left(\Phi_{O F F}\right)-\omega_{-}\right] / g \sim 3$, and is well reproduced by theory. The largest discrepancy is observed for pulse amplitudes $\Delta \Phi \sim 90 m \Phi_{0}$, where additional features are clearly seen in the experiment. We attribute them to a small density of NV centers having a ${ }^{13} \mathrm{C}$ among their closest neighbor, known to shift the electron spin frequency by $\pm 65 \mathrm{MHz}$ due to the hyperfine interaction, and that was not taken into account in the calculation.

We finally perform a Ramsey-like experiment in order to quantify the time during which the microwave field can be stored in the spin ensemble. Starting as before with a steady-state microwave field in the resonator biased at $\Phi_{\text {OFF }}$, the resonator is tuned in resonance with the spins at $t=0$ for a $\pi / 2$ pulse $\left(g_{\text {ens }} \tau=\pi / 2\right)$, after which it is detuned by $\Delta \omega / 2 \pi=30 \mathrm{MHz}$ during $\Delta t$, then tuned back to resonance for a second $\pi / 2$ pulse, and finally tuned back at $\omega_{\mathrm{r}}\left(\Phi_{\mathrm{OFF}}\right)$, after which $a(\Delta t)=\overline{A\left(2 \tau+\Delta t+t_{\mathrm{off}}\right)}$ is measured. Due to the beating between the effective spin oscillator and the resonator, oscillations are observed in $a(\Delta t)$ at frequency $\Delta \omega$ as seen in Fig,4. The oscillations amplitude decay time $\sim 30$ ns gives the storage time, on the order of $\sim 2 / \Delta$ as expected. The full calculation, performed as explained above, yields again reasonable agreement with the measurements.

As shown by the previous results, inhomogeneous broadening appears as a serious obstacle to the successful implementation of hybrid quantum circuits based on spin ensembles. One first obvious solution is to obtain samples with narrower inhomogeneous linewidths. In the specific case of NV centers, this requires a very efficient conversion rate $\eta$ of $\mathrm{P} 1$ centers initially present in the diamond crystal in NV centers. Improvement by one order of magnitude compared to the sample used in this work (where $\eta$ is a few percent) is within reach. This would reduce the inhomogeneous linewidth by the same amount, at which point inhomogeneous broadening would be dominated by the NV centers hyperfine structure $(\Delta / 2 \pi \sim 4 \mathrm{MHz}$ for $\left.{ }^{14} N\right)$. Another solution is to increase the NV concentration even further to reach the regime $g_{\text {ens }} \gg \Delta$ and rely on the cavity protection effect. Finally, it should be possible, by applying refocusing techniques used for atomic ensemble-based quantum memories [21], to recover the quantum information lost in the dark states into the superradiant state and then into the resonator. This would be the best way to fully benefit from the spin superior coherence properties.

In conclusion we have demonstrated the coherent storage and retrieval of a classical microwave field of $\sim 100$ photons from a superconducting resonator into collective excitations of an ensemble of NV centers and discussed the implications of inhomogeneous broadening. This work opens the way to the operation of a quantum memory in which a single microwave photon would be coherently stored and retrieved from a superconducting qubit into the spin ensemble.

We gratefully thank P. Sénat, J.-C. Tack, P.-F. Orfila, M. de Combarieu, P. Forget, and P. Pari for priceless technical help, and we acknowledge useful discussions within the Quantronics group. We acknowledge the support of European Contracts MIDAS and SOLID.
[1] H. Paik et al., arxiv:cond-mat/1105.4652 (2011).

[2] C.F. Roos et al., Phys. Rev. Lett. 92, 220402 (2004).

[3] G. Balasubramanian et al., Nature Mat. 8, 383 (2009).

[4] P. Rabl et al., Phys. Rev. Lett. 97, 033003 (2006).

[5] D. Petrosyan et al., Phys. Rev. A 79, 040304(R) (2009).

[6] J. Verdu et al., Phys. Rev. Lett 103, 043603 (2009).

[7] A. Imamoglu, Phys. Rev. Lett. 102, 083602 (2009).

[8] J.H. Wesenberg et al., Phys. Rev. Lett. 103, 070502 (2009).

[9] Y. Kubo et al., Phys. Rev. Lett. 105, 140502 (2010).

[10] D.I. Schuster et al., Phys. Rev. Lett. 105, 140501 (2010).

[11] R. Amsuss et al., Phys. Rev. Lett. 107, 060502 (2011).
[12] P. Bushev et al., Phys. Rev. B 84, 060501 (2011)

[13] H. Wu et al., Phys. Rev. Lett. 105, 140503 (2010).

[14] Z. Kurucz, J.H. Wesenberg and K. Molmer, Phys. Rev. A 83, 053852 (2011).

[15] I. Diniz et al., arxiv:quant-ph/1101.1842 (2011).

[16] A. Palacios-Laloy et al., J. Low Temp. Phys. 151, 1034 (2008).

[17] M. Sandberg et al., Appl. Phys. Lett. 92, 203501 (2008).

[18] F.R. Ong et al., Phys. Rev. Lett. 106, 167002 (2011).

[19] J.A. van Wyk et al., J. Phys. D 30, 1790 (1997).

[20] V.V. Dobrovitski, A.E. Feiguin, D.D. Awschalom and R. Hanson, Phys. Rev. B 77, 245212 (2008). 
[21] A.I. Lvovsky, B.C. Sanders and W. Tittel, Nature Phot. 3, 706 (2009). 


\section{Supplementary Material}

\section{MEASUREMENT SETUP}

Figure 5(a) shows the full configuration of the room temperature apparatus, and 5(b) the wiring inside the fridge. The fast current pulse is generated by an arbitrary function generator AFG3251 (Tektronix) and input into the fridge from the port "Flux in" through a $10 \mathrm{~dB}$ attenuator. After being filtered by low pass filters (LPF1 and LPF2) at each temperature stage and further attenuated by $20 \mathrm{~dB}$ at $4 \mathrm{~K}$ stage, the flux pulse passes through the flux line [see the solid arrows in Fig. [6(a) and (b)], and goes back to room temperature through low-pass filters (LPF3 and LPF4) and a $20 \mathrm{~dB}$ attenuator. The end of the fast flux circuit ("Flux out") is terminated by a broadband $50 \Omega$.

The microwave pulse is generated by mixing continuous microwave and a fast DC voltage pulse using an IQ-mixer 1]. The microwave pulse is input to the port "Reso in" after being attenuated by a tunable attenuator, and reaches to the input port of the chip through attenuators at each temperature stage and a band-pass pass filter (BPF1) at $40 \mathrm{mK}$. After being filtered by a band pass filter (BPF2) and isolated by two circulators, the transmitted signal is amplified by a cryogenic HEMT (high electron mobility transistor) amplifier having noise temperature $\sim 4 \mathrm{~K}$. The amplified signal is then further amplified at room temperature by two microwave amplifiers, and demodulated into in-(I) and quadrature-(Q) phase components using an IQ-demodulator. The demodulated signal is sampled at $500 \mathrm{MS} / \mathrm{s}$ by a fast data acquisition card Acqiris CC103 (Agilent) after being further amplified by two amplifiers.

The resonator chip fixed onto a printed circuit board is mounted inside a sample box made of copper [Fig. 5(b)]. A DC magnetic field $B_{N V}$ is applied parallel to the chip by passing a DC current through an outer superconducting coil. The sample box and the coil are surrounded by two magnetic shieldings consisting of permalloy tape VC6025X (VacuumSchmelze) and a lead cylinder [not shown in Fig. 5(b)].

\section{FLUX PULSE}

Here we describe a few technical details on the flux pulse used to tune the resonator frequency at the nanosecond scale. As shown in Fig. 6, the pulse is applied using an arbitrary function generator AFG3252 (Tektronix) that sends a current pulse through an on-chip line. The risetime of the voltage pulse is 2 ns. Passing current through the on-chip antenna generates screening currents through the ground planes, as shown by the dashed arrows in Fig. 6(b), of our superconducting circuit with very low damping times (of the order of the ms) that in turn affect the flux through the SQUID. As a result, the SQUID offset flux bias point was found to depend on the time integral of the flux pulse. In addition, the flux applied to the SQUID loop by sending a fast current pulse through the flux line was strongly reduced (by a factor $\sim 50$ ) compared to the flux applied with a dc current of the same amount. We measure this current-to-flux transfer function with calibration experiments, allowing us to convert a voltage pulse into flux as in figure 3 of the main text. In order for the bias point to be independent of the amplitude and duration of the pulse, we had to add, at the end of each sequence, a compensation flux pulse opposite to the first one [see Fig. 6(c)]. Such a compensation pulse has strictly no incidence on the experiment outcome since it is applied long after the microwave signal is detected.

\section{THEORY DETAILS}

We now give more details on the theory calculations. All these calculations are performed in the HolsteinPrimakoff approximation, in which the spins and the resonator are described by harmonic oscillators, as explained in the text. The system Hamiltonian is $H / \hbar=$ $\omega_{\mathrm{r}}(\Phi) a^{\dagger} a+\sum \omega_{\mathrm{j}} b_{\mathrm{j}}^{\dagger} b_{\mathrm{j}}+\sum i g_{\mathrm{j}}\left(b_{\mathrm{j}}^{\dagger} a-b_{\mathrm{j}} a^{\dagger}\right), g_{\mathrm{j}}$ being the coupling constant of spin $j$ with the resonator.

\section{A. Rabi oscillations}

We first calculate the oscillating signal resulting from the cycles of storage and retrieval of the resonator field of amplitude $\alpha$ into the spin ensemble (fig. $2 \mathrm{~d}$ in the article). A first remark is that the system consisting of coupled harmonic oscillators, its time evolution does not depend on the initial field amplitude. As a result we only calculate $\alpha_{R a b i}=\left\langle 0\left|a(t) a^{\dagger}(0)\right| 0\right\rangle$ which represents the probability amplitude that a photon created at $t=0$ is still present at time $t$. As shown in 2 this quantity can be calculated by considering an effective non-Hermitian Hamiltonian

$$
H_{e f f} / \hbar=\left(\begin{array}{cccc}
\tilde{\omega}_{0} & i g_{1} & i g_{2} & \ldots \\
-i g_{1} & \tilde{\omega}_{1} & & \\
-i g_{2} & & \tilde{\omega}_{2} & \\
\vdots & & & \ddots
\end{array}\right) .
$$

with complex angular frequencies $\tilde{\omega}_{0}=\omega_{r}-i \kappa / 2$ and $\tilde{\omega}_{k}=\omega_{k}-i \gamma_{0} / 2$; here, $\gamma_{0}$ is the spontaneous emission rate of each spin (that we take here to be zero since NV centers at low temperature have negligible energy relaxation). Indeed, introducing the vector $X(t)$ of coordinates $\left[\left\langle a(t) a^{\dagger}(0)\right\rangle, \ldots,\left\langle b_{j}(t) a^{\dagger}(0)\right\rangle, \ldots\right]$ it can be shown that $d X / d t=-(i / \hbar) H_{\text {eff }} X$. The formal solution to this equation is then

$$
X(t)=\mathcal{L}^{-1}\left[\left(s+i H_{e f f} / \hbar\right)^{-1} X(0)\right],
$$


(a)

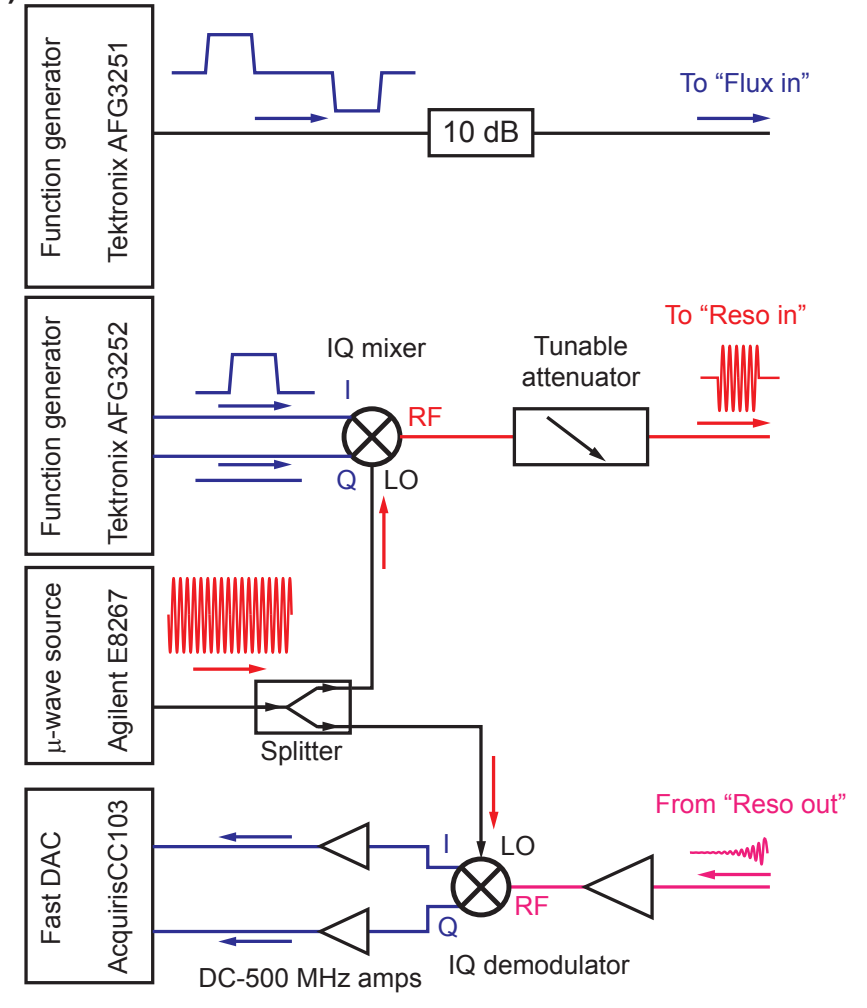

(b)

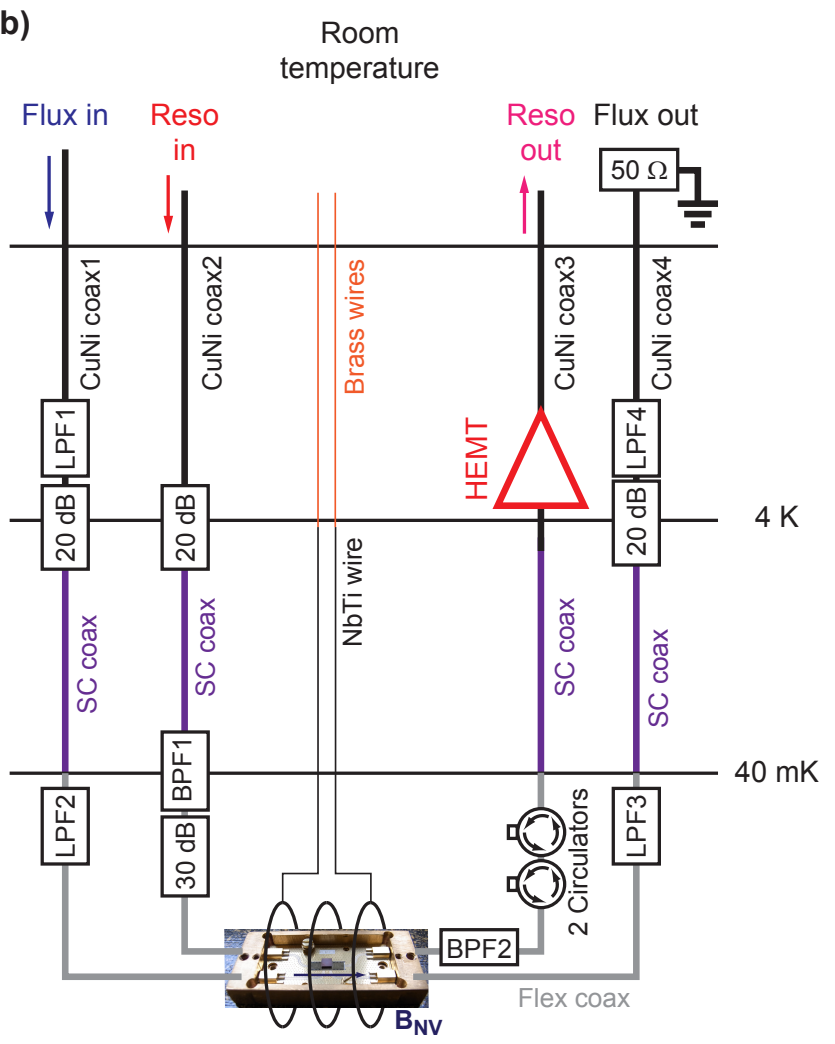

Figure 5: Measurement setup and wiring. (a) Full configuration of the measurement apparatus at room temperature. (b) Schematic of the wiring inside the dilusion refrigerator. LPF1, LPF2, LPF3, and LPF4 are low-pass filters having cutoff frequencies $1.35 \mathrm{GHz}, 450 \mathrm{MHz}, 450 \mathrm{MHz}$, and $1.35 \mathrm{GHz}$, respectively. BPF1 actually consists of two series of low-pass (5.4 $\mathrm{GHz}$ ) and high-pass $(1.3 \mathrm{GHz})$ filters, and BPF2 is a band-pass filter (2.5 to $4 \mathrm{GHz})$. CuNi coax1, 2, and 4 are coaxial cables made of $\mathrm{CuNi}$, and $\mathrm{CuNi}$ coax3 is a silver-plated $\mathrm{CuNi}$ coaxial cable. $\mathrm{SC}$ coax is a superconducting NbTi coaxial cable. Flex coax is a low-loss flexible coaxial cable. The two circulators are STE-1502KS (Pamtech). Here the small rectangles represent ports terminated by $50 \Omega$. HEMT is a cryogenic microwave amplifier having low-noise temprature (Caltech). A DC current for the DC magnetic field $B_{N V}$ is applied through an RC low-pass filter at room temperature.

with $X(0)=x_{G}$ and $x_{G} \equiv(1,0, \ldots, 0)$. This implies that $\alpha(t)=x_{G}^{\dagger} \cdot X(t)=\mathcal{L}^{-1}\left[t_{1}(s)\right]$ with $t_{1}(s)=x_{G}^{\dagger} \cdot\left(s+i H_{e f f}\right)^{-1} \cdot x_{G}$ and $\mathcal{L}[f(s)]=$ $\int e^{-s t} f(t) d t, s$ being a complex number. Since $t_{1}(s)$ is not singular on its imaginary axis, we only need $t_{1}$ for pure imaginary argument $s=-i \omega$ to perfom the Laplace transform inversion. As shown in [2, we have $t_{1}(-i \omega)=i /\left[\omega-\omega_{0}+i \kappa / 2-W(\omega)\right]$ with $W(\omega)=g_{\text {ens }}^{2} \int \rho\left(\omega^{\prime}\right) d \omega^{\prime} /\left[\omega-\omega^{\prime}+i \gamma_{0} / 2\right]$. Computing $\alpha(t)$ is thus achieved by evaluating $t_{1}$ for the distribution $\rho(\omega)$ described in the main text, and numerically evaluting the inverse Laplace transform. This calculation also enables us to calculate the transmission as plotted in Fig.1e, because as shown in [2], the resonator transmission is obtained as $t(\omega)=-(\kappa / 2) t_{1}(-i \omega)$.

An additional complication in the experiment is that the resonator-spin detuning $\delta$ during the microwave pulse is finite, implying that the initial state is actually already hybridized. To take this into account in the calculation, we make the approximation that this initial state is a coherent superposition of $x_{G}$ and $x_{S} \equiv$ $\left(0, g_{1}, \ldots, g_{j}, . ., g_{N}\right) / g_{e n s}$. The vector $x_{s}$ is associated with the excitation of the superradiant mode. Thus the initial state is written $x(t=0)=\cos (\theta / 2) x_{G}+i \sin (\theta / 2) x_{S}$ with mixing angle $\tan \theta=2 g_{\text {ens }} / \delta$. Using a similar analysis, we now have $\alpha_{R a b i}=\cos (\theta / 2) \mathcal{L}^{-1}\left[t_{1}(s)\right]+$ $i \sin (\theta / 2) \mathcal{L}^{-1}\left[t_{4}(s)\right]$. In addition to $t_{1}(s)$ we then need $t_{4}(s)=x_{G}^{\dagger}\left(s+i H_{e f f}\right)^{-1} x_{S}$, as shown in [2], $t_{4}(-i \omega)=$ $-i t_{1}(-i \omega) W(\omega) / g_{\text {ens }}$.

\section{B. Ramsey fringes}

Each $\pi / 2$ pulse necessary to realize the Ramsey-like experiment is realised by bringing the resonator and spins to resonance. For a fast pulse, the resonant interaction maps continuously the coherent state of the field to the superradiant mode in the spins. We calibrate the interaction time in such a way as to transform the state $x_{G}$ into the superposition $\frac{x_{G}-x_{S}}{\sqrt{2}}$. After the first $\pi / 2$ pulse, the resonator is kept detuned from the spin ensemble for a time $t$. The system state at this point can be evaluated using eq. (2). We define $X_{G}(t)\left(\operatorname{resp} . X_{S}(t)\right)$ as the 


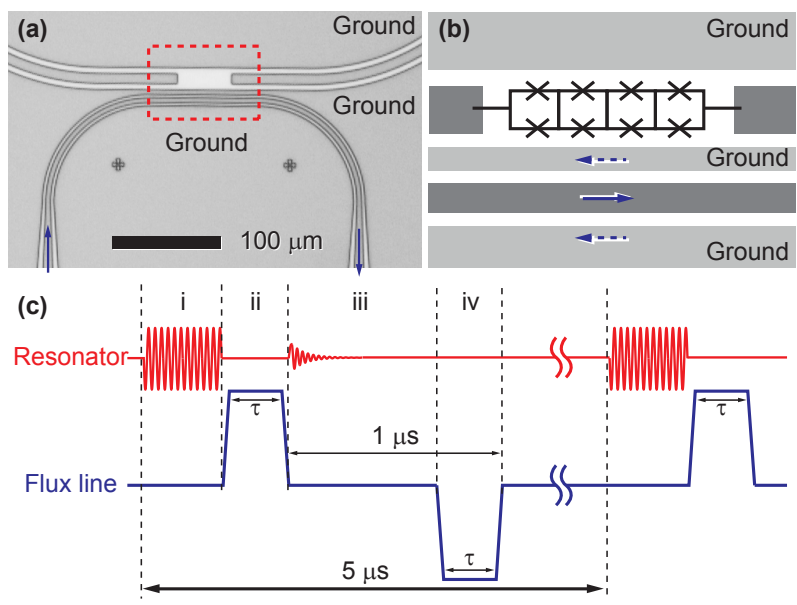

Figure 6: (a) Photograph of the area where the 4-SQUIDs array is placed in the resonator. Note that this photograph was taken before the SQUIDs were fabricated. The arrows shows a flow of an applied fast current pulse. (b) Schematic of inside the dashed box in (a) (here the SQUIDs are drawn). The applied (return) current pulses is represented by a solid (dashed) arrow(s). (c) Actual pulse sequence for the measurement: (i) excitation, (ii) interaction, (iii) data accumulation, and (iv) compensation in the sequence. After data sampling (iii), an inverted pulse (iv) is applied to compensate the time integral to 0 (see text). The whole length of one sequence is $5 \mu \mathrm{s}$.

vector of coordinates $\left[\left\langle a(t) a^{\dagger}(0)\right\rangle, \ldots,\left\langle b_{j}(t) a^{\dagger}(0)\right\rangle, \ldots\right]$ at time $t$ with initial conditions $x_{G}$ (resp. $\left.x_{S}\right)$. A second $\pi / 2$ pulse is then applied before the amplitude $\alpha_{R F}$ of the field in the resonator is measured :

$$
\begin{aligned}
\alpha_{R F} & =\frac{1}{\sqrt{2}} x_{G}^{\dagger} \cdot U_{\pi / 2}\left(X_{G}(t)-X_{S}(t)\right) \\
& =\frac{1}{2}\left(x_{G}^{\dagger}+x_{S}^{\dagger}\right) \cdot\left(X_{G}(t)-X_{S}(t)\right) \\
& =\frac{1}{2} \mathcal{L}^{-1}\left(t_{1}(s)-t_{2}(s)+t_{3}(s)-t_{4}(s)\right),
\end{aligned}
$$

where $t_{2}(s)=x_{S}^{\dagger} \cdot\left(s+i H_{e f f}\right)^{-1} \cdot x_{S}$ and $t_{2}(s)=x_{S}{ }^{\dagger}$. $\left(s+i H_{e f f}\right)^{-1} \cdot x_{G}, t_{1}$ and $t_{4}$ are defined above. As shown in [2], $t_{2}(-i \omega)=-t_{1}(-i \omega) W(\omega)\left(s+i \tilde{\omega}_{0}\right) / g_{\text {ens }}^{2}$ and $t_{3}=$ $-t_{4}$.

\section{Density distribution}

The spins density distribution that we use is the convolution of a normalized Gaussian of standard deviation $\sigma$ with a normalized Lorentzian of HWHM $\gamma$. The resulting distribution is known as the Voigt profile and is also normalized.

$$
\begin{aligned}
\rho\left(\omega^{\prime} ; \sigma, \gamma\right) & =\int_{-\infty}^{\infty} G\left(\omega^{\prime \prime} ; \sigma\right) L\left(\omega^{\prime}-\omega^{\prime \prime} ; \gamma\right) d \omega^{\prime} \\
& =\frac{1}{\sigma \sqrt{2 \pi}} \frac{\gamma}{\pi} \int_{-\infty}^{\infty} \frac{e^{\omega^{\prime \prime 2} / 2 \sigma^{2}}}{\left(\omega^{\prime}-\omega^{\prime \prime}\right)^{2}+\gamma^{2}} d \omega^{\prime}
\end{aligned}
$$

An important property is that we can compute analytically the function $W(\omega)$ :

$W\left(\omega ; \sigma, \gamma, \gamma_{0}\right)=\frac{\Omega^{2}}{\sigma \sqrt{2 \pi}} \frac{\gamma}{\pi} \iint \frac{e^{\omega^{\prime \prime 2} / 2 \sigma^{2}}}{\left(\omega^{\prime}-\omega^{\prime \prime}\right)^{2}+\gamma^{2}} \frac{d \omega^{\prime} d \omega^{\prime \prime}}{\omega-\omega^{\prime}+i \gamma_{0}}$

Taking the limit $\gamma_{0} \rightarrow 0$ we have

$W(\omega ; \sigma, \gamma)=-i \sqrt{\frac{\pi}{2}} \frac{\Omega^{2}}{\sigma} \exp \left\{-\left(\frac{\omega+i \gamma}{\sigma \sqrt{2}}\right)^{2}\right\} \operatorname{erfc}\left(-i \frac{\omega+i \gamma}{\sigma \sqrt{2}}\right)$

The function that is taken in the theory plots of the main text actually takes into account the well-known NV center hyperfine splitting due to ${ }^{14} N$ by adding up three identical distributions $\rho(\omega)$ separated by $2.2 \mathrm{MHz}$ [3]. The resulting distribution is the one shown in figure 2 of the main text. This is conviently done since $W\left[\rho_{1}+\rho_{2}\right]=$ $W\left[\rho_{1}\right]+W\left[\rho_{2}\right]$ for any two different spin distributions $\rho_{1,2}$. Using this formula for $W(\omega)$ we can evaluate the functions $t_{i}$ necessary for the simulation of the Rabi and Ramsey-like experiments.
[1] Here a DC constant voltage is applied to the Q-port of the IQ-mixer for the perpose of cancelling the offset, i.e., to prevent the microwave signal from leaking out during the pulse is off.
[2] I. Diniz et al., arxiv:quant-ph/1101.1842 (2011).

[3] V. Acosta et al., Phys. Rev. B 80, 115202 (2009). 